\title{
ORIGINAL ARTICLE High sensitivity blood-based M-protein detection in sCR patients with multiple myeloma
}

\author{
JR Mills ${ }^{1}$, DR Barnidge ${ }^{1}$, A Dispenzieri ${ }^{1,2}$ and DL Murray ${ }^{1}$
}

We assessed the ability of a mass spectrometry-based technique, called monoclonal immunoglobulin rapid accurate mass measurement (miRAMM), to extend the analytical range of M-protein detection in serum samples obtained from myeloma patients in stringent complete response (sCR) post-autologous stem cell transplant (ASCT). To aid the M-protein detection post ASCT, the accurate molecular mass of the M-protein light chain at diagnosis was determined in all patients $(N=30)$ and used to positively identify clones in the sCR serum. Day 100 post-ASCT, sCR samples had miRAMM identifiable M-proteins in $81 \%$ of patients. Patients who had achieved only a partial remission (PR) pre-ASCT and those with lgG isotypes serum samples had the highest rate of M-protein detection by miRAMM. miRAMM positivity at single time points (day 100, 6 months or 12 months) did not correlate with progression-free survival (PFS). In contrast, sCR patients who did not decrease their miRAMM M-protein intensities in serial measurements had shorter PFS than those whose miRAMM intensities decreased (median 17.9 months vs 51.6 months; $P<0.0017$ ). miRAMM M-protein is a more sensitive blood-based test than traditional M-protein tests and could cost effectively aid in serially monitoring complete remission for continue response or biochemical relapse.

Blood Cancer Journal (2017) 7, e590; doi:10.1038/bcj.2017.75; published online 25 August 2017

\section{INTRODUCTION}

As the number of effective therapies for treatment of multiple myeloma continues to increase, a greater proportion of patients are achieving deep therapeutic responses. ${ }^{1-3}$ Traditionally, serum and urine M-protein measurements by immunoelectrophoresis were sufficient to define treatment responses in $90 \%$ of cases. ${ }^{4}$ However, as more patients have deeper treatment responses, current M-protein detection methods are analytically incapable of monitoring patient deep response. Achieving and maintaining complete remission (CR) is important as it translates into longer periods of progression-free survival (PFS) and overall survival ${ }^{5,6}$ but even among CR patients there is significant variability in the duration of overall survival. ${ }^{7}$ Therefore, in order to further stratify patients who achieve $C R$, more sensitive methods for detecting minimal residual disease (MRD) have been developed. To increase the analytical sensitivity, the focus has shifted towards analyzing the bone marrow (BM) for MRD. Two clinical BM MRD approaches have emerged: molecular-based methods detecting clonal variable diverse joining heavy chain rearrangements ${ }^{8}$ and multicolor flow cytometry ${ }^{9}$ for detecting malignant clonal plasma cells. BM molecular-based methods for MRD are $>3$ orders of magnitude more sensitive than serum M-protein detection by immunofixation (IFE) and free light chain (FLC). ${ }^{10}$ These more sensitive BMbased MRD methods are superior predictors of PFS. Unfortunately, patients classified as MRD negative continue to experience disease progression. It is possible that BM MRD methods may have sampling biases due to the patchy nature of BM infiltration. ${ }^{11}$ Therefore, there is still need for a test to monitor stringent complete response $(s C R)$ patients for disease recurrence. Serial sampling using BM has drawbacks in terms of overall expense and patient discomfort. Therefore, a less invasive, cost effective test from blood may be useful in monitoring SCR patients.

Low-level quantification of a single immunoglobulin (lg) M-protein clone in the midst of a polyclonal lg background consisting of hundreds of thousand similar lg molecules is both a daunting and intriguing proposition. Two mass spectrometric (MS) methods have been developed to extend M-protein quantification to lower levels. One method is based on the detection of unique tryptic peptides corresponding to the heavy and light chain variable region of an M-protein, which has been termed the 'clonotypic' peptide approach, ${ }^{12-14}$ and a second method, termed 'miRAMM' (monoclonal Ig rapid accurate mass measurements), which is based on identification of the M-protein from the accurate molecular mass of light chain component. ${ }^{15}$ Both methods take advantage of the fact that each M-protein undergoes a specific recombination event generating a unique variable domain and thus a unique molecular mass. Both methods provide accurate quantitative information with limits of detection that are 100 times lower than that of IFE. ${ }^{14,15}$ The improvements in detection limits arises from superior resolution of accurate mass measurements to separate the M-protein from the polyclonal background in comparison with electrophoretic protein charge measurements. The miRAMM method has the intrinsic advantage of simplicity allowing for adaption to the clinical lab with shorter turnaround times than the clonotypic method. The clonotypic approach, however, is less dependent on the M-protein being expressed above the polyclonal background but is more prone to interference by low-level complementary determining region tryptic peptides from the endogenous polyclonal background as the M-protein level decreases.

\footnotetext{
${ }^{1}$ Protein Immunology Laboratory, Department of Laboratory Medicine and Pathology, Mayo Clinic, Rochester, MN, USA and ${ }^{2}$ Division of Hematology, Department of Medicine, Mayo Clinic, Rochester, MN, USA. Correspondence: Dr DL Murray, Protein Immunology Laboratory, Department of Laboratory Medicine and Pathology, Mayo Clinic, 200 First Street Southwest, Rochester, MN, 55905, USA.

E-mail: murray.david@mayo.edu

Received 21 March 2017; revised 29 June 2017; accepted 5 July 2017
} 
The miRAMM method original described using liquid chromatography (LC)-coupled electrospray ionization-time-of-flight (TOF)-based MS platforms has also been adapted to a lower cost chromatography-free matrix assisted laser desorption/ionization (MALDI)-TOF MS platform with performance properties suited for the high volume clinical lab. ${ }^{16}$ Analytical times are rapid ( $<1$ minute per patient) and when combined with five separate immunoprecipitations for $\lg G, \lg A, \lg M, k$ and $\lambda$, the method can detect, quantitate and isotype M-proteins. The analytical equipment (Bruker, Microflex MALDI-TOF, Billerica, MA, USA) has also gained acceptance in the clinical lab for identification of bacteria. Recent application of MALDI-TOF MS method to patients with established clinical diagnosis demonstrated that MALDI-TOF MS had comparable clinical sensitivity with protein electrophoresis/ IFE/FLC methods. ${ }^{17}$ However, although MALDI-TOF MS methods are quick and robust, electrospray ionization-TOF instruments provide superior resolving power and mass accuracy enabling a lower limit of detection. Thus, electrospray ionization-TOF miRAMM is better suited for quantitating low level M-proteins and resolving potential t-mAb drug interferences.

In this study, we have assessed the ability of a high sensitivity MS-based miRAMM method to extend the analytical range of traditional M-protein measurements in patients who in patients who achieve stringent $\mathrm{CR}(\mathrm{s} C \mathrm{R})$ post autologous stem cell transplant (ASCT).

\section{MATERIALS AND METHODS}

\section{Nanobody enrichment}

Serum Ig enrichment was performed using a 50:50 mix of camelid-derived nanobodies directed against the LC constant domains of $\mathrm{K}$ and $\lambda$ (Thermo Fisher Scientific PN: 084910 and 083310), Life Technologies, Carlsbad, CA USA. Briefly, $10 \mu$ l of beads were incubated with $20 \mu \mathrm{l}$ of serum diluted into $180 \mu \mathrm{l}$ of phosphate-buffered saline for $45 \mathrm{~min}$ at ambient temperature. Subsequently, the supernatant was removed and the beads were washed three times with $200 \mu$ l of phosphate-buffered saline and then twice with $200 \mu \mathrm{l}$ of water. Samples were eluted with $20 \mu \mathrm{l}$ of $5 \%$ acetic acid containing $50 \mathrm{~mm}$ tris [2-carboxyethyl] phosphine, to disassociate lgs into separated LC and heavy chain components.

\section{Liquid chromatography}

An Eksigent Ekspert 200 microLC (Foster City, CA, USA) was used to separate $\mathrm{lg}$ LCs before ionization and detection. The mobile phases included an aqueous phase A ( $100 \%$ water $+0.1 \%$ formic acid) and an organic phase B ( $90 \%$ acetonitrile $+10 \%$ isopropanol $+0.1 \%$ formic acid). Two microlitres of each bead elution was injected per analysis onto a Poroshell 300SB-C3 column $(1.0 \mathrm{~mm} \times 75 \mathrm{~mm})$ with a $5 \mu \mathrm{m}$ particle size placed in a $60^{\circ} \mathrm{C}$ column heater. The gradient used has been described previously and the flow rate was $25 \mu \mathrm{l} / \mathrm{min}^{15}$

\section{Mass spectrometry}

A SCIEX TripleTOF 5600 quadrupole TOF MS using electrospray ionization in positive ion mode was used for miRAMM analysis. Source conditions have been described previously. ${ }^{15}$ Data analysis was performed using Analyst TF v1.6 and PeakView ver. 2.2 (AbSciex, Framingham, MA, USA). The mass spectra of the multiply charged LC ions were deconvoluted to accurate molecular mass using the Bio Tool Kit ver. 2.2 plug-in software (Asb Sciex, Framingham, MA, USA). The retention time of the monoclonal LC in each pre-treatment patient sample was tracked using PeakView. Subsequent detection of residual monoclonal LCs in post-treatment samples was performed by searching for the presence of a monoclonal LC above the polyclonal background with a molecular mass within $\pm 1 \mathrm{Da}$ of pretreatment monoclonal LC with a retention time within $0.5 \mathrm{~min}$ of that identified in the pre-treatment sample. The LC signal intensity was recorded as counts per seconds. The instrument was calibrated every five samples using the automated calibrant delivery system. Mass measurement accuracy was estimated to be 15 p.p.m. over the course of the analysis.

\section{Patient samples}

The Mayo Foundation Institutional Review Board approved this retrospective study. All patients gave written informed consent to have their medical records reviewed. Patient selection criteria included: availability of a serum sample within 30 days of initial diagnosis at Mayo Clinic Rochester from 2005 to 2012 and between 3 and 12 months post-ASCT, and attainment of a SCR using serum and urine IFE, serum Ig FLC and BM 6-color multicolor flow cytometry with a sensitivity of $10^{-4}$ to $10^{-5}$ within

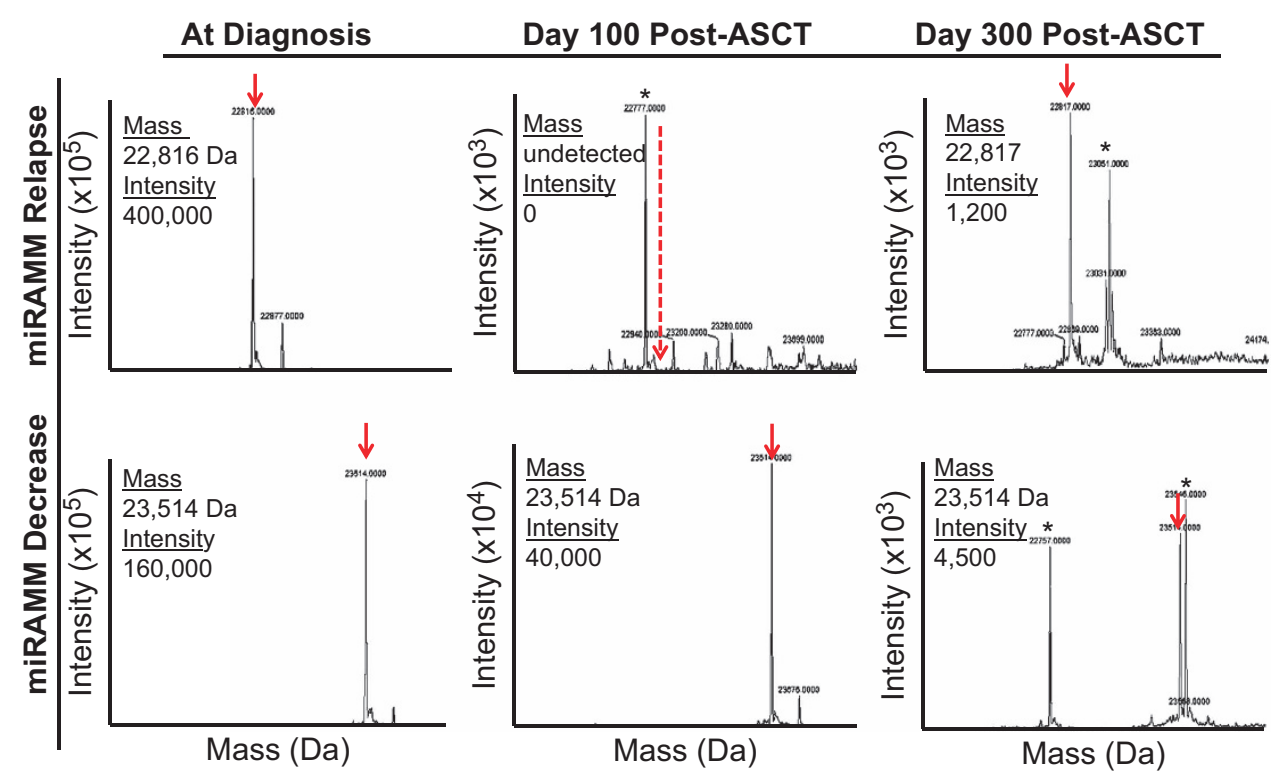

Figure 1. A deepening miRAMM response is suggestive of longer PFS in patients in SCR. Examples of miRAMM data. The top panel indicates a patient who had a miRAMM relapsed. At diagnosis the monoclonal LC mass signature was readily identifiable (red arrow). This mass signature was then assessed in subsequent post-treatment samples. At day 100, there is undetectable disease by miRAMM (dashed red arrow). The astrisk indicates a secondary oligoclonal response. However, 200 days later there MS signature of this patients disease had re-emerged indicating a relapse (red arrow). The bottom panel indicates a patient with a deepening miRAMM response. Between diagnosis and 100 days post-ASCT, the MS signature for this patient decreased from an intensity of 160000 to 40000 . Approximately 200 days later, this has continued to decline and is now at 4500 . 


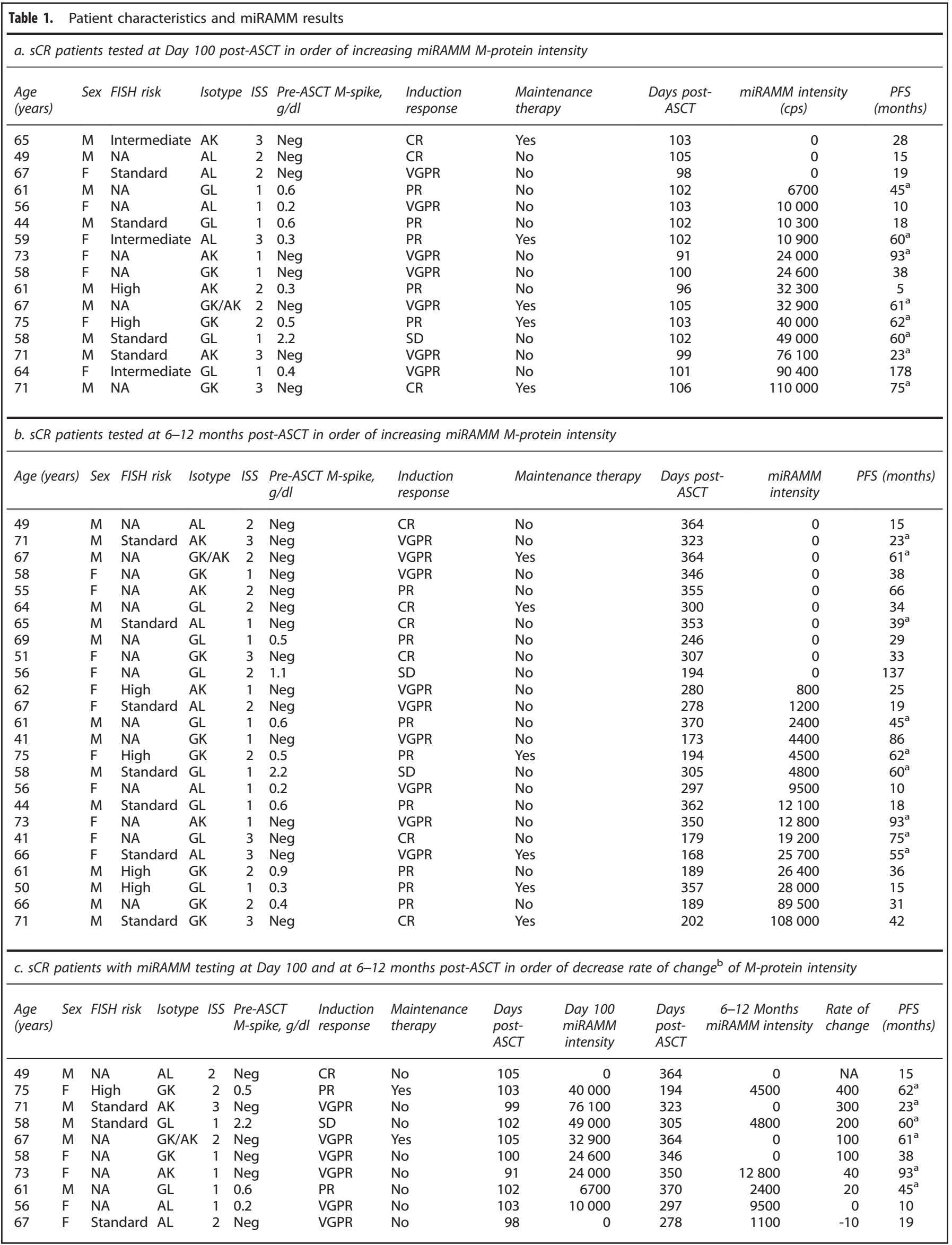


Table 1. (Continued)

c. SCR patients with miRAMM testing at Day 100 and at 6-12 months post-ASCT in order of decrease rate of change of M-protein intensity

\begin{tabular}{|c|c|c|c|c|c|c|c|c|c|c|c|c|c|}
\hline $\begin{array}{l}\text { Age } \\
\text { (years) }\end{array}$ & Sex & FISH risk & Isotype & ISS & $\begin{array}{l}\text { Pre-ASCT } \\
\text { M-spike, g/dl }\end{array}$ & $\begin{array}{l}\text { Induction } \\
\text { response }\end{array}$ & $\begin{array}{l}\text { Maintenance } \\
\text { therapy }\end{array}$ & $\begin{array}{l}\text { Days } \\
\text { post- } \\
\text { ASCT }\end{array}$ & $\begin{array}{l}\text { Day } 100 \\
\text { miRAMM } \\
\text { intensity }\end{array}$ & $\begin{array}{l}\text { Days } \\
\text { post- } \\
\text { ASCT }\end{array}$ & $\begin{array}{l}\text { 6-12 Months } \\
\text { miRAMM intensity }\end{array}$ & $\begin{array}{l}\text { Rate of } \\
\text { change }\end{array}$ & $\begin{array}{c}\text { PFS } \\
\text { (months) }\end{array}$ \\
\hline 44 & $M$ & Standard & $\mathrm{GL}$ & 1 & 0.6 & PR & No & 102 & 10300 & 362 & 12100 & -10 & 18 \\
\hline
\end{tabular}

Abbreviations: AK, IgA kappa; AL, IgA lambda; $\mathrm{ASCT}$, autologous stem cell transplant; $\mathrm{cps}$, counts per seconds; $\mathrm{CR}$, complete remission; $\mathrm{F}$, female; $\mathrm{FISH}$, fluorescence in situ hybridization; GK, IgG kappa; GL, IgG lambda; ISS, International Staging System; M, male; miRAMM, monoclonal immunoglobulin rapid accurate mass measurement; NA, not applicable; Neg, negative; PFS, progression-free survival; PR, partial remission; SCR, stringent complete response; SD, stable diseas; VGPR, very good partial remission. ${ }^{a}$ Censored data, these patients remain disease-free at the time of writing this manuscript. ${ }^{\mathrm{b}} \mathrm{A}$ rate of change in miRAMM M-protein intensity was calculated by dividing the change in miRAMM intensity by the time interval between measurements in days.

120 days of ASCT. Thirty patients from the Mayo Clinic dysproteinemia sample bank fulfilled these criteria. A subset of these patients with durable CR had two stored serum samples in this 3-12 months interval.

\section{RESULTS}

The accurate molecular mass of the M-protein LC was readily identified (100\% applicability) in all 30 diagnostic serum samples and served as a surrogate of M-protein identification in subsequent analysis (Figure 1). The use of a high-resolution MS capable of achieving a mass measurement accuracy ( $<15$ p.p.m.) enabled the distinction of any LC with at least a 1 Da difference in mass. This mass accuracy was essential for evaluating post-ASCT serum samples as it was common for these samples to contain several 'M-spikes' in the LC mass distribution (Figure 1). Thus, to establish an M-spike in a post-ASCT sample as related to the original M-protein, the LC mass had to match the original peak within $\pm 1 \mathrm{Da}$ and the LC retention time within $\pm 30 \mathrm{~s}$ of the diagnostic sample.

As per selection criteria for this study, all patients were documented in the electronic medical record to have achieved sCR at day 100 using conventional established testing criteria. ${ }^{10}$ All post-ASCT serum samples evaluated in this study were negative by serum protein electrophoresis, IFE and FLC assay. Sixteen of 30 patients had samples available for miRAMM testing collected when SCR was established at day 100 post-ASCT ( \pm 9 days). Of these samples, miRAMM identified the presence of the M-protein in $81 \%$ (13 of 16$)$ of the patients (Table 1a). The three, day 100 miRAMM-negative cases were all $\lg A$ and were negative by serum protein electrophoresis pre-ASCT; surprisingly, these patients did not have prolonged PFS in comparison to the cohort. Twenty-five patients had serum samples available for testing by miRAMM that were collected between 6 and 12 months post-ASCT where SCR was maintained. Of these samples, $60 \%$ (15 of 25 ) were positive by miRAMM for M-protein (Table 1b). Patients who achieved CR preASCT were more likely to be miRAMM negative 6-12 months postASCT as compared with those who did not (67\% (4 of 6) and $32 \%$ (6 of 19), respectively). At these later time points, IgG and $\lg A$ isotypes were equally likely to be miRAMM negative (38\% (6 of 16) versus $44 \%$ (4 out of 9)). In this small data set, single time points (day 100 or 6-12 months) miRAMM M-protein positivity status did not predict for better PFS.

The data were then re-examined in regards to the change in M-protein intensity between the two time points (Table 1c). Eleven ${ }^{11}$ patients had serum samples available at two different sampling dates (day 100 plus $\sim 6-12$ months) during which they remained in SCR. A rate of change in M-protein intensity was calculated by dividing the change in miRAMM intensity by the time interval between measurements in days. A continuing decrease in the miRAMM M-protein intensity (a positive rate of change) over time would be expected to correlate with a longer PFS, whereas failure to decrease or increasing intensities (a negative rate of change) should correlate with a shorter PFS. Of the 11 patients, 3 had converted to miRAMM-negative status at the second sampling, 1 had maintained miRAMM-negative status, 4 had a further reduction in miRAMM M-protein intensity ( $>40 \%$ from day 100 to the second sampling), 2 miRAMM M-proteins of stable intensity and 1 converted from miRAMM negative to miRAMM positive. The eight patients with continued miRAMM negativity or decreasing miRAMM M-protein intensity had longer PFS compared with those who had stable or increasing miRAMM M-protein intensity (51.6 months vs 17.9 months, respectively $P<0.0017$ ).

\section{DISCUSSION}

The data from this sCR cohort support previous studies, indicating that miRAMM is a more analytically sensitive method to detect M-proteins than electrophoresis. ${ }^{15}$ Although being more sensitive, the detection of M-protein at a single time point did not correlate with PFS for this relatively small non-homogeneous cohort. A confounding factor to in our data is the half-life of M-protein in blood (average 21-25 days for $\lg G$ and 7-14 days for $\lg A$ ), which would cause a time lag between tumor lysis and M-protein decrease; an effect which becomes more pronounce at lower total Ig concentrations. ${ }^{18}$ Patients with $\lg \mathrm{A}$ M-proteins and lower M-proteins concentrations pre-ASCT would need a shorter time interval to clear circulating M-protein from blood than $\lg G$ M-proteins with high levels pre-ASCT. This was supported by the day 100 results in which the three miRAMM-negative patients were all $\lg A$ patients (shorter half-life than $\operatorname{lgG}$ ) with nonquantifiable M-protein pre-ASCT. By $\sim 12$ months, IgA and IgG were equally as likely to be miRAMM negative. Therefore, the timing of the evaluation by this method, or any sensitive blood method, needs to be carefully considered. Although Ig recycling can delay the clearance of the M-protein, it cannot cause the $M$-protein to increase or remain constant. Hence, the change in serial miRAMM M-protein measurements can aid in detecting active M-protein production. Thus, the finding of this study demonstrating that increases in the miRAMM M-protein intensity were correlated with shorter PFS is not surprising. miRAMM is well suited for serial sampling due to its cost-effective, less-invasive nature in comparison with BM aspiration. Further work in this area should focus on defining the biological and analytic variability of low level miRAMM M-proteins so that the clinical significance of changes in miRAMM M-protein intensity can be accurately defined. The results of this study highlight the recent recommendations from the IMWG consensus criteria for response and MRD in multiple myeloma. ${ }^{10}$ In their recommendation, the group states that the development of peripheral blood-based monitoring should be the ultimate goal as it would allow for serial sampling without the trauma of repeated BM aspirations and assure complete eradication of the tumor by assessment of the extramedullary compartment which is not evaluated by BM biopsy. 
BM MRD status provides potentially important information not achievable by this method such as tumor clone evolution, cellular immune system reconstitution and direct detection of plasma cells. BM MRD is also not confounded by lg half-lives. Hence, we do not see this method as substitute for BM MRD but rather as method to follow CR patients or as a companion method to accurately time BM MRD measurements. We acknowledge that miRAMM M-protein measurements may be subject to same limitations as other M-protein measurements such as in nonsecretory myeloma. Other limitations of this study include a small sample size, limited follow-up, and inclusion of only patients with intact M-proteins rather than including patients with Bence Jones myeloma. Despite these limitations, our study provides compelling preliminary evidence that this simple MS-based test can serve as an assay that extends the current value of monitoring M-proteins beyond that of FLC, serum and urine IFE, and that serial sampling of patients can provide information on active M-protein production. Future studies evaluating the miRAMM method should also consider serial sampling with absolute quantification by miRAMM as opposed to the relative concentrations.

\section{CONFLICT OF INTEREST}

JRM, DRB and DLM have intellectual property rights filed on the method. AD declares no conflict of interest.

\section{ACKNOWLEDGEMENTS}

We thank Tara Phelps for overseeing collection and management of patient samples.

\section{AUTHOR CONTRIBUTIONS}

JRM, DLM and AD designed the study. AD and JRM collected and reviewed clinical data. DRB, JRM and DLM developed the assay utilized in the study. JRM and DRB performed miRAMM analysis. All authors prepared the final manuscript.

\section{REFERENCES}

1 Jakubowiak AJ, Dytfeld D, Griffith KA, Lebovic D, Vesole $D H$, Jagannath $\mathrm{S}$ et al. A phase $1 / 2$ study of carfilzomib in combination with lenalidomide and low-dose dexamethasone as a frontline treatment for multiple myeloma. Blood 2012; 120 : 1801-1809.

2 Sonneveld P, Goldschmidt H, Rosinol L, Blade J, Lahuerta JJ, Cavo M et al. Bortezomib-based versus nonbortezomib-based induction treatment before autologous stem-cell transplantation in patients with previously untreated multiple myeloma: a meta-analysis of phase III randomized, controlled trials. J Clin Oncol 2013; 31: 3279-3287.

3 Cavo M, Tacchetti P, Patriarca F, Petrucci MT, Pantani L, Galli M et al. Bortezomib with thalidomide plus dexamethasone compared with thalidomide plus dexamethasone as induction therapy before, and consolidation therapy after, double autologous stem-cell transplantation in newly diagnosed multiple myeloma: a randomised phase 3 study. Lancet 2010; 376: 2075-2085.
4 Chee CE, Kumar S, Larson DR, Kyle RA, Dispenzieri A, Gertz MA et al. The importance of bone marrow examination in determining complete response to therapy in patients with multiple myeloma. Blood 2009; 114: 2617-2618.

5 Gonsalves WI, Gertz MA, Dispenzieri A, Lacy MQ, Lin Y, Singh PP et al. Implications of continued response after autologous stem cell transplantation for multiple myeloma. Blood 2013; 122: 1746-1749.

6 Martinez-Lopez J, Blade J, Mateos MV, Grande C, Alegre A, Garcia-Larana J et al. Long-term prognostic significance of response in multiple myeloma after stem cell transplantation. Blood 2011; 118: 529-534.

7 Harousseau JL, Attal M, Avet-Loiseau H. The role of complete response in multiple myeloma. Blood 2009; 114: 3139-3146.

8 Martinez-Lopez J, Lahuerta JJ, Pepin F, Gonzalez M, Barrio S, Ayala R et al. Prognostic value of deep sequencing method for minimal residual disease detection in multiple myeloma. Blood 2014; 123: 3073-3079.

9 Rawstron AC, Child JA, de Tute RM, Davies FE, Gregory WM, Bell SE et al. Minimal residual disease assessed by multiparameter flow cytometry in multiple myeloma: impact on outcome in the Medical Research Council Myeloma IX Study. J Clin Oncol 2013; 31: 2540-2547.

10 Kumar S, Paiva B, Anderson KC, Durie B, Landgren O, Moreau P et al. International Myeloma Working Group consensus criteria for response and minimal residual disease assessment in multiple myeloma. Lancet Oncol 2016; 17: e328-e346.

11 Paiva B, Puig N, Garcia-Sanz R, San Miguel JF. Is this the time to introduce minimal residual disease in multiple myeloma clinical practice? Clin Cancer Res 2015; 21: 2001-2008.

12 Barnidge DR, Tschumper RC, Theis JD, Snyder MR, Jelinek DF, Katzmann JA et al. Monitoring M-proteins in patients with multiple myeloma using heavy-chain variable region clonotypic peptides and LC-MS/MS. J Proteome Res 2014; 13: 1905-1910.

13 Remily-Wood ER, Benson K, Baz RC, Chen YA, Hussein M, Hartley-Brown MA et al. Quantification of peptides from immunoglobulin constant and variable regions by LC-MRM MS for assessment of multiple myeloma patients. Proteomics Clin Appl 2014; 8: 783-795.

14 Bergen HR 3rd, Dasari S, Dispenzieri A, Mills JR, Ramirez-Alvarado M, Tschumper RC et al. Clonotypic light chain peptides identified for monitoring minimal residual disease in multiple myeloma without bone marrow aspiration. Clin Chem 2016; 62: 243-251.

15 Barnidge DR, Dasari S, Botz CM, Murray DH, Snyder MR, Katzmann JA et al. Using mass spectrometry to monitor monoclonal immunoglobulins in patients with a monoclonal gammopathy. J Proteome Res 2014; 13: 1419-1427.

16 Mills JR, Kohlhagen MC, Dasari S, Vanderboom PM, Kyle RA, Katzmann JA et al. Comprehensive assessment of M-proteins using nanobody enrichment coupled to MALDI-TOF mass spectrometry. Clin Chem 2016; 62: 1334-1344.

17 Milani P, Murray DL, Barnidge DR, Kohlhagen MC, Mills JR, Merlini G et al. The utility of MASS-FIX to detect and monitor monoclonal proteins in the clinic. Am J Hematol 2017; 92: 772-779.

18 Kendrick F, Evans ND, Arnulf B, Avet-Loiseau H, Decaux O, Dejoie T et al. Analysis of a compartmental model of endogenous immunoglobulin $\mathrm{G}$ metabolism with application to multiple myeloma. Front Physiol 2017; 8: 149.

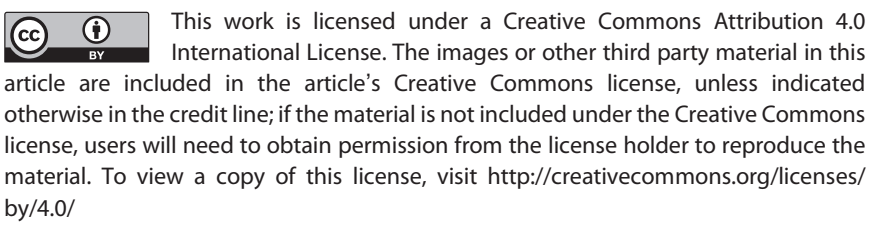
by/4.0/

(c) The Author(s) 2017 